\title{
Appendix: Pathology Supplement
}

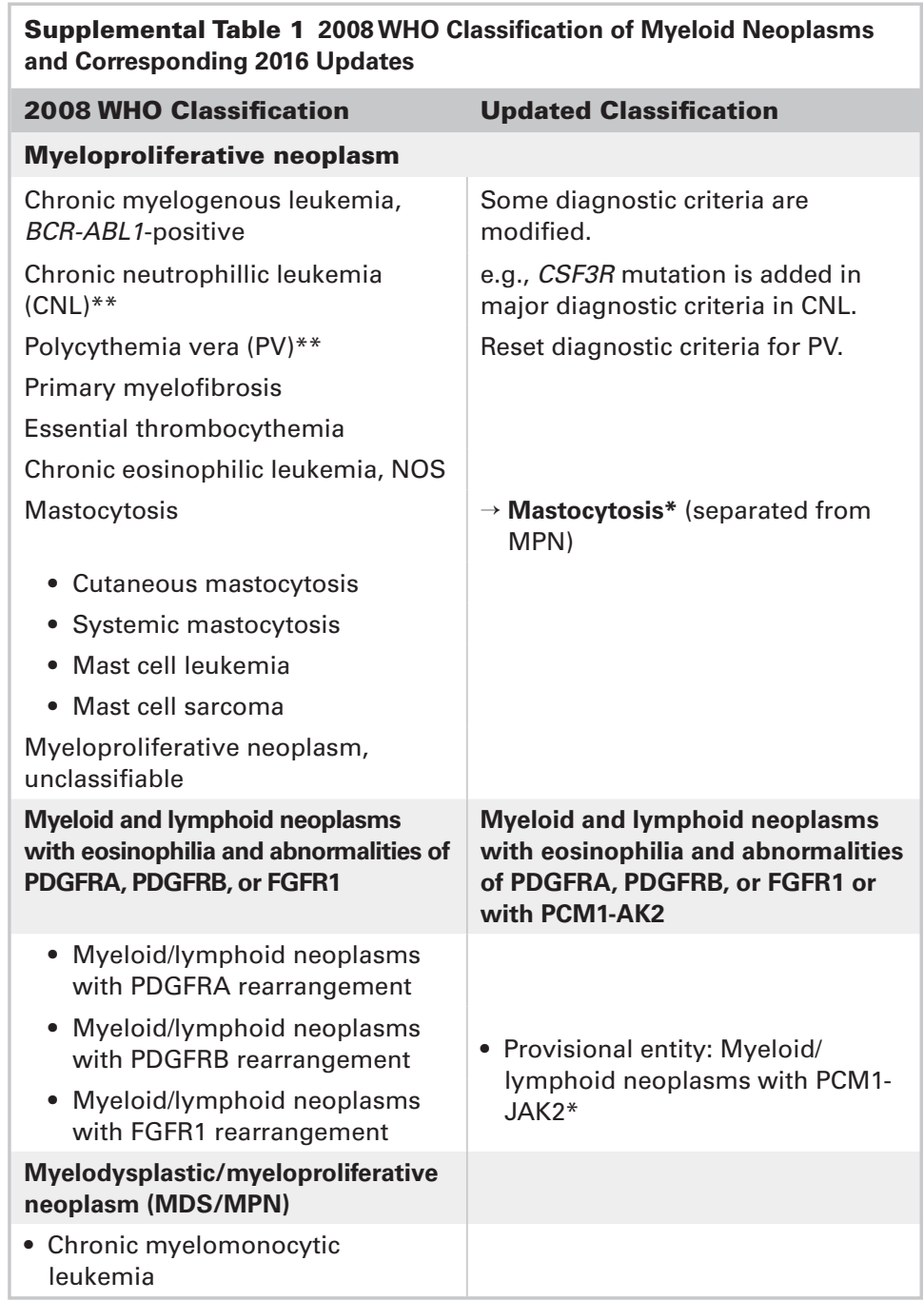

(continued) 


\section{Supplemental Table 12008 WHO Classification of Myeloid Neoplasms and Corresponding 2016 Updates (continued)}

\section{WHO Classification}

- Atypical chronic myeloid leukemia, $B C R-A B L 1$ negative

- Juvenile myelomonocytic leukemia

- Myelodysplastic/myeloproliferative neoplasm, unclassifiable

- Provisional entity: refractory anemia with ring-sideroblasts with associated thrombocytosis (RARS-T)

\section{Myelodysplastic syndromes}

- Refractory cytopenia with unilieage dysplasia

- Refractory anemia with ring sideroblasts

- Refractory cytopenia with multilineage dysplasia

- Refractory anemia with excess blasts

- Myelodysplastic syndrome with isolated $\operatorname{del}(5 q)$

- Myelodysplastic syndrome, unclassifiable

- Provisional entity: refractory cytopenia of childhood

\section{Acute myeloid leukemia and} related neoplasms

- $A M L$ with $\mathrm{t}(8 ; 21)(\mathrm{q} 22 ; 21)$ (q22;q22.1); RUNX1-RUNX1T1

- $A M L$ with inv(16)(p13;p13.1q22) or $\mathrm{t}(16 ; 16)(\mathrm{p} 13.1 ; \mathrm{q} 22)$; CBFB-MYH11

\section{Updated Classification}

$\rightarrow$ Myelodysplastic/myeloproliferative neoplasm with ring sieroblasts and thrombocytosis (MDS/ MPN-RS-T)**

$\rightarrow$ MDS with single-lineage dysplasia**

$\rightarrow$ MDS with ring sideroblasts**

- MDS with ring sideroblasts and single-lineage dysplasia**

- MDS with ring sideroblasts with multilineage dysplasia**

$\rightarrow$ MDS with multilineage dysplasia**

$\rightarrow$ MDS with excess blasts**

$\rightarrow$ MDS with isolated del $(5 q) * *$

$\rightarrow$ MDS, unclassifiable**

$\rightarrow$ The same

Myeloid neoplasms with germline predisposition* 


\section{Supplemental Table 12008 WHO Classification of Myeloid Neoplasms and Corresponding 2016 Updates (continued)}

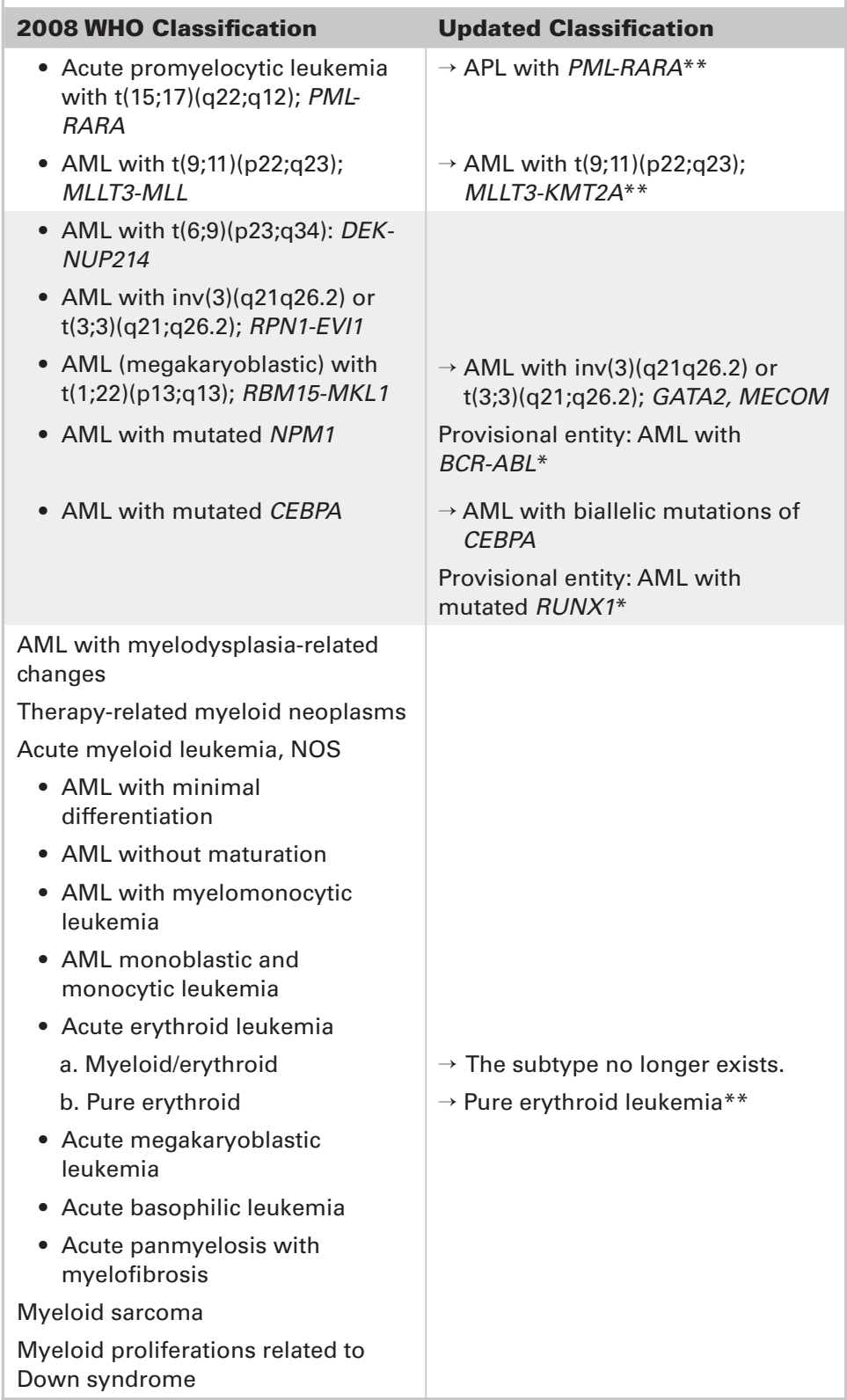




\section{Supplemental Table 12008 WHO Classification of Myeloid Neoplasms and Corresponding 2016 Updates (continued)}

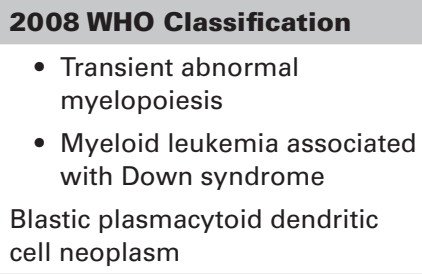

- Transient abnormal myelopoiesis

- Myeloid leukemia associated with Down syndrome

Blastic plasmacytoid dendritic cell neoplasm

\section{Acute leukemias of ambiguous} lineage

Acute undifferentiated leukemia

Mixed phenotype acute leukemia with $\mathrm{t}(9 ; 22)(\mathrm{q} 34 ; \mathrm{q} 11.2) ; B C R-A B L 1$

Mixed phenotype acute leukemia with $\mathrm{t}(\mathrm{v} ; 11 \mathrm{q} 23) ; M L L$ rearranged

Mixed phenotype acute leukemia, B/ myeloid, NOS

Mixed phenotype acute leukemia, T/ myeloid, NOS

Mixed phenotype acute leukemia, NOS - rare

Other ambiguous lineage leukemia

- Natural killer (NK) - cell lymphoblastic leukemia/ lymphoma

\section{B lymphoblastic leukemia/} lymphoma

B lymphoblastic leukemia/ lymphoma, NOS

B lymphoblastic leukemia/ lymphoma with recurrent cytogenetic abnormalities

- B lymphoblastic leukemia/lymphoma with $\mathrm{t}(9 ; 22)$ (q34;q11.2); $B C R-A B L 1$

- B lymphoblastic leukemia/lymphoma with $\mathrm{t}(\mathrm{v} ; 11 \mathrm{q} 23.3)$; MLL rearranged

- B lymphoblastic leukemia/ lymphoma with $\mathrm{t}(12 ; 21)(\mathrm{p} 13$; q22); TEL-AML1(ETV6-RUNX1)

$\rightarrow$ No longer in AML category; please see T-lymphoblastic leukemia/lymphoma $\rightarrow$ B lymphoblastic leukemia/lymphoma with $\mathrm{t}(\mathrm{v} ; 11 \mathrm{q} 23.3) ; K M T 2 A$ rearranged 


\section{Supplemental Table 12008 WHO Classification of Myeloid Neoplasms and Corresponding 2016 Updates (continued)}

\section{WHO Classification}

- B lymphoblastic leukemia/lymphoma with hyperdiploidy

- B lymphoblastic leukemia/ lymphoma with hypodiploidy (Hypodiploid ALL)

- B lymphoblastic leukemia/lymphoma with $\mathrm{t}(5 ; 14)(\mathrm{q} 31 ; \mathrm{q} 32)$; IL3-lgH

- B lymphoblastic leukemia/ lymphoma with $\mathrm{t}(1 ; 19)(\mathrm{q} 23$; p13.3); E2A-PBX1(TCF3-PBX1)

\section{Updated Classification}

Provisional entity:

B lymphoblastic leukemia/ lymphoma, BCR-ABL1-like*

Provisional entity:

B lymphoblastic leukemia/ lymphoma with iAMP21*

\section{T-lymphoblastic leukemia/} lymphoma

\section{Provisional entity: early T-cell precursor lymphoblastic leukemia \\ Provisional entity: natural killer cell lymphoblastic leukemia/ lymphoma}

*Newly proposed myeloid neoplasms listed in the revision of $2008 \mathrm{WHO}$ classification, including those provisional lymphomas in 2008.

**The diagnostic criteria of myeloid neoplasms are modified in the revision of 2008 WHO classification. 


\section{Supplemental Table 22008 WHO Classification of Mature B-Cell, T-Cell, and NK-Cell Neoplasms and Corresponding 2016 Updates}

\section{WHO Classification \\ Updated Classification}

\section{Mature B-cell neoplasms}

Chronic lymphocytic leukemia/small lymphocytic lymphoma

B-cell prolymphocytic leukemia Splenic marginal zone lymphoma Hairy cell leukemia Splenic lymphoma/leukemia, unclassifiable

- Splenic diffuse red pulp small B-cell lymphoma

- Hairy cell leukemia variant

Lymphoplasmacytic lymphoma

Waldenström macroglobulinemia Heavy chain diseases

- Alpha heavy chain disease

- Gamma heavy chain disease

- Mu heavy chain disease

Plasma cell myeloma

Solitary plasmacytoma of bone

Extraosseous plasmacytoma

Extranodal marginal zone lymphoma of mucosaassociated lymphoid tissue (MALT lymphoma)

Nodal marginal zone lymphoma

- Pediatric nodal marginal zone lymphoma*

Follicular lymphoma

Pediatric follicular lymphoma*

Primary cutaneous follicle center lymphoma rearrangement*
Monoclonal B-cell lymphocytosis*

Monoclonal gammopathy of undetermined significance (MGUS), Ig $\mathrm{M}^{*}$ Monoclonal gammopathy of undetermined significance (MGUS), $\lg \mathrm{G} / \lg \mathrm{A}^{*}$

Monoclonal immunoglobulin deposition disease*

In situ follicular lymphoma*

Duodenal follicular lymphoma*

Large B-cell lymphoma with IRF4 


\begin{tabular}{|c|c|}
\hline $\begin{array}{l}\text { Supplemental Table } 22008 \text { WHO C } \\
\text { and NK-Cell Neoplasms and Corresp }\end{array}$ & $\begin{array}{l}\text { lassification of Mature B-Cell, T-Cell, } \\
\text { nding } 2016 \text { Updates (continued) }\end{array}$ \\
\hline 2008 WHO Classification & Updated Classification \\
\hline & $\begin{array}{l}\text { In situ mantle cell lymphoma* } \\
\text { Germinal center B-cell type* } \\
\text { Activated B-cell type* }\end{array}$ \\
\hline Mantle cell lymphoma & \\
\hline $\begin{array}{l}\text { Diffuse large B-cell lymphoma } \\
\text { (DLBCL), NOS }\end{array}$ & \\
\hline $\begin{array}{l}\text { - T-cell/histiocyte rich large } \\
\text { B-cell lymphoma }\end{array}$ & \\
\hline - Primary DLBCL of the CNS & \\
\hline $\begin{array}{l}\text { - Primary cutaneous DLBCL, } \\
\text { leg type }\end{array}$ & \\
\hline $\begin{array}{l}\text { EBV positive DLBCL of the } \\
\text { elderly** }\end{array}$ & $\begin{array}{l}\text { EBV positive DLBCL, NOS } \\
\text { (not limited in elderly)* }\end{array}$ \\
\hline $\begin{array}{l}\text { DLBCL associated with chronic } \\
\text { inflammation }\end{array}$ & EBV positive mucocutaneous ulcer* \\
\hline Lymphomatoid granulomatosis & \\
\hline $\begin{array}{l}\text { Primary mediastinal (thymic) large } \\
\text { B-cell lymphoma }\end{array}$ & \\
\hline Intravascular large B-cell lymphoma & \\
\hline ALK Positive large B-cell lymphoma & \\
\hline Plasmablastic lymphoma & \\
\hline Primary effusion lymphoma & \\
\hline $\begin{array}{l}\text { Large B-cell lymphoma arising in } \\
\text { HHV8-associated multicentric Cas- } \\
\text { tleman disease }^{* *}\end{array}$ & HHV8 positive DLBCL* \\
\hline Burkitt lymphoma & $\begin{array}{l}\text { Burkitt-like lymphoma with } \\
\text { 11q aberration* }\end{array}$ \\
\hline $\begin{array}{l}\text { B-cell lymphoma, unclassifiable, with } \\
\text { features intermediate between diffuse } \\
\text { large B-cell lymphoma and Burkitt } \\
\text { lymphoma** }\end{array}$ & $\begin{array}{l}\text { High-grade B-cell lymphoma } \\
\text { with MYC and BCL-2 and/or } \\
\text { BCL-6 rearrangements* } \\
\text { High-grade B-cell lymphoma, NOS* }\end{array}$ \\
\hline $\begin{array}{l}\text { B-cell lymphoma, unclassifiable, } \\
\text { with features intermediate between } \\
\text { diffuse large B-cell lymphoma and } \\
\text { classical Hodgkin lymphoma }\end{array}$ & \\
\hline
\end{tabular}




\section{Supplemental Table 22008 WHO Classification of Mature B-Cell, T-Cell, and NK-Cell Neoplasms and Corresponding 2016 Updates (continued)}

2008 WHO Classification
Mature T- and NK-cell neoplasms
T-cell prolymphocytic leukemia
T-cell large granular
lymphocytic leukemia
Chronic lymphoproliferative
disorder of NK cell
Aggressive NK-cell leukemia
Systemic EBV+T-cell lymphoprolife
ative disease of childhood*
Hydroa vacciniforme-like
lymphoma*
AdultT-cell leukemia/lymphoma
Extranodal NK-/T-cell lymphoma,
nasal type
Enteropathy-associatedT-cell
lymphoma

Hepatosplenic T-cell lymphoma

Subcutaneous panniculitis-like T-cell lymphoma

Mycosis fungoides

Sézary syndrome

Primary cutaneous CD30+T-cell lymphoproliferative disorders

- Lymphomatoid papulosis

- Primary cutaneous anaplastic large cell lymphoma

Primary cutaneous gamma-delta T-cell lymphoma

Primary cutaneous CD8+ aggressive epidermotropic cytotoxic T-cell lymphoma
Updated Classification

Monomorphic epitheliotropic intestinal T-cell lymphoma* Indolent T-cell lymphoproliferativedisorder of the gastrointestinal (GI) tract*
Primary cutaneous acral CD8+T-cell lymphoma* 


\begin{tabular}{|c|c|}
\hline $\begin{array}{l}\text { Supplemental Table } 22008 \text { WHO C } \\
\text { and NK-Cell Neoplasms and Correspc }\end{array}$ & $\begin{array}{l}\text { assification of Mature B-Cell, T-Cell, } \\
\text { nding } 2016 \text { Updates (continued) }\end{array}$ \\
\hline 2008 WHO Classification & Updated Classification \\
\hline $\begin{array}{l}\text { Primary cutaneous CD4+ small/ } \\
\text { medium T-cell lymphoma }\end{array}$ & \\
\hline PeripheralT-cell lymphoma, NOS & FollicularT-cell lymphoma* \\
\hline $\begin{array}{l}\text { Angioimmunoblastic T-cell lympho- } \\
\text { ma }\end{array}$ & $\begin{array}{l}\text { Nodal peripheral T-cell lymphoma } \\
\text { with TFH phenotype }\end{array}$ \\
\hline $\begin{array}{l}\text { Anaplastic large cell lymphoma, ALK } \\
\text { positive }\end{array}$ & \\
\hline $\begin{array}{l}\text { Anaplastic large cell lymphoma, ALK } \\
\text { negative* }\end{array}$ & \\
\hline & $\begin{array}{l}\text { Breast implant-associated anaplastic } \\
\text { large cell lymphoma* }\end{array}$ \\
\hline $\begin{array}{l}\text { Post-transplant lymphoproliferative } \\
\text { disorders (PTLD) }\end{array}$ & \\
\hline $\begin{array}{l}\text { Plasmacytic hyperplasia } \\
\text { Infectious mononucleosis-like PTLD } \\
\text { Polymorphic PTLD }\end{array}$ & \\
\hline $\begin{array}{l}\text { Monomorphic PTLD (B-andT-/ } \\
\text { NK-cell types) }\end{array}$ & Florid follicular hyperplasia PTLD \\
\hline $\begin{array}{l}\text { Classical Hodgkin lymphoma } \\
\text { type PTLD }\end{array}$ & \\
\hline Hodgkin lymphoma & \\
\hline $\begin{array}{l}\text { Nodular lymphocyte predominant } \\
\text { Hodgkin lymphoma }\end{array}$ & \\
\hline $\begin{array}{l}\text { Classical Hodgkin lymphoma } \\
\text { - Nodular sclerosis } \\
\text { - Mixed cellularity } \\
\text { - Lymphocyte-rich } \\
\text { - Lymphocyte-deplete }\end{array}$ & \\
\hline $\begin{array}{l}\text { *Newly proposed lymphomas listed in } t \\
\text { including those provisional lymphomas }\end{array}$ & $\begin{array}{l}\text { he revision of } 2008 \text { WHO classification, } \\
\text { n } 2008 .\end{array}$ \\
\hline $\begin{array}{l}\text { **The types of lymphomas are modifiec } \\
\text { classification. }\end{array}$ & in the revision of $2008 \mathrm{WHO}$ \\
\hline
\end{tabular}




\section{Supplemental Table 3 Key Gene Mutations That Predict Overall Patient Prognosis in Myeloid and Lymphoid Neoplasms}

\begin{tabular}{|c|c|c|c|c|}
\hline $\begin{array}{l}\text { Gene } \\
\text { Name }\end{array}$ & Location & Protein Class & $\begin{array}{l}\text { Associated } \\
\text { Disease(s) }\end{array}$ & $\begin{array}{l}\text { Prognostic } \\
\text { Impact }\end{array}$ \\
\hline $\begin{array}{l}\text { FLT3/ITD } \\
\text { FLT3/TKD }\end{array}$ & $13 q 12.2$ & $\begin{array}{l}\text { Growth factor } \\
\text { receptor tyrosine } \\
\text { kinase } \\
\text { Signal } \\
\text { transduction }\end{array}$ & MDS, AML & $\begin{array}{l}\text { - Adverse } \\
\text { - None in } \\
\text { APL }\end{array}$ \\
\hline NPM1 & $5 q 35.1$ & $\begin{array}{l}\text { Nuclear and } \\
\text { cytoplasmic } \\
\text { protein shuttling }\end{array}$ & AML, ALCL & $\begin{array}{l}\text { Favorable } \\
\text { (in absence } \\
\text { or low } \\
\text { allelic ratio } \\
\text { of FLT3/ITD) } \\
\text { Favorable in } \\
\text { ALCL }\end{array}$ \\
\hline$M L L$ & $11 q 23$ & $\begin{array}{l}\text { Transcriptional } \\
\text { regulation } \\
\text { Histone } \\
\text { methyltransferase }\end{array}$ & MDS, AML & Adverse \\
\hline CEBPA & $19 q 13.1$ & $\begin{array}{l}\text { Transcriptional } \\
\text { regulation }\end{array}$ & AML & $\begin{array}{l}\text { Favorable, } \\
\text { when bial- } \\
\text { lelic CEBPA } \\
\text { mutated }\end{array}$ \\
\hline TET2 & $4 q 24$ & DNA methylation & MDS, AML & Neutral \\
\hline DNMT3A & $2 p 23$ & DNA methylation & MDS, AML & Neutral \\
\hline$I D H 1 / 2$ & $\begin{array}{l}2 p 33.3 \text { and } \\
15 q 26.1\end{array}$ & DNA methylation & $\begin{array}{l}\text { MDS, MPN, } \\
\text { AML }\end{array}$ & $\begin{array}{l}\text { Neutral/ } \\
\text { Adverse }\end{array}$ \\
\hline$A S X L 1$ & $20 q 11$ & $\begin{array}{l}\text { Chromatin/histone } \\
\text { modification }\end{array}$ & $\begin{array}{l}\text { MDS, MDS/ } \\
\text { MPN }\end{array}$ & Adverse \\
\hline$E Z H 2$ & $7 q 35-q 36$ & $\begin{array}{l}\text { Chromatin/histone } \\
\text { modification }\end{array}$ & $\begin{array}{l}\text { MDS, MDS/ } \\
\text { MPN }\end{array}$ & Adverse \\
\hline$R U N X 1$ & $21 q 22.3$ & $\begin{array}{l}\text { Transcriptional } \\
\text { regulation }\end{array}$ & MDS, AML & Adverse \\
\hline $\begin{array}{l}\text { BCOR/ } \\
\text { BCORL1 }\end{array}$ & Xp11.4 & $\begin{array}{l}\text { Transcriptional } \\
\text { regulation }\end{array}$ & MDS, AML & Adverse \\
\hline ETV6 & $12 \mathrm{p} 13$ & $\begin{array}{l}\text { Transcriptional } \\
\text { regulation }\end{array}$ & MDS, AML & Adverse \\
\hline SETBP1 & $18 q 12.3$ & $\begin{array}{l}\text { Transcriptional } \\
\text { regulation }\end{array}$ & $\begin{array}{l}\text { MDS, MDS/ } \\
\text { MPN }\end{array}$ & Adverse \\
\hline TP53 & $17 p 13.1$ & $\begin{array}{l}\text { DNA repair/tumor } \\
\text { suppressor }\end{array}$ & $\begin{array}{l}\text { Various } \\
\text { myeloid and } \\
\text { lymphoid } \\
\text { malignancies }\end{array}$ & Adverse \\
\hline
\end{tabular}




\begin{tabular}{|c|c|c|c|c|}
\hline \multicolumn{5}{|c|}{$\begin{array}{l}\text { Supplemental Table } 3 \text { Key Gene Mutations That Predict Overall } \\
\text { Patient Prognosis in Myeloid and Lymphoid Neoplasms (continued) }\end{array}$} \\
\hline $\begin{array}{l}\text { Gene } \\
\text { Name }\end{array}$ & Location & Protein Class & $\begin{array}{l}\text { Associated } \\
\text { Disease(s) }\end{array}$ & $\begin{array}{l}\text { Prognostic } \\
\text { Impact }\end{array}$ \\
\hline JAK2 & $\begin{array}{l}9 p 24.1 \\
\text { V617F } \\
\text { codon }\end{array}$ & $\begin{array}{l}\text { Signal } \\
\text { transduction }\end{array}$ & MPN, MDS & Neutral \\
\hline$C B L$ & $11 q 23.3$ & $\begin{array}{l}\text { Signal } \\
\text { transduction }\end{array}$ & $\begin{array}{l}\text { MDS, MDS/ } \\
\text { MPN }\end{array}$ & Adverse \\
\hline SRSF2 & $17 q 25.1$ & RNA splicing & MDS & Adverse \\
\hline SF3B1 & $2 q 33.1$ & RNA splicing & MDS, CLL & $\begin{array}{l}\text { Favorable } \\
\text { (MDS) }\end{array}$ \\
\hline MYD88 & $3 p 22$ & NF-kB activation & $\begin{array}{l}\text { LPL/WM } \\
\text { (>90\%), } \\
\text { CLL (10\%), } \\
\text { DLBCL, ABC } \\
\text { type }(8 \%)\end{array}$ & $\begin{array}{l}\text { Adverse in } \\
\text { DLBCL }\end{array}$ \\
\hline ATM & $11 q 23-q 23$ & $\begin{array}{l}\text { DNA repair } \\
\text { Tumor } \\
\text { suppressor }\end{array}$ & $\begin{array}{l}\text { CLL/SLL, } \\
\text { MCL, T-PLL }\end{array}$ & Adverse \\
\hline$B R A F$ & $\begin{array}{l}\text { V600E } \\
\text { codon }\end{array}$ & $\begin{array}{l}\text { Signal } \\
\text { transduction }\end{array}$ & $\begin{array}{l}\text { Hairy cell } \\
\text { leukemia }\end{array}$ & $\begin{array}{l}\text { Driver } \\
\text { mutation, } \\
\text { may respond } \\
\text { to BRAF } \\
\text { inhibitors }\end{array}$ \\
\hline \multicolumn{5}{|c|}{$\begin{array}{l}\text { ALCL, anaplastic large; } \mathrm{AML} \text {, acute myeloid leukemia; } \mathrm{CLL} \text {, chronic } \\
\text { lymphocytic leukemia; ITD, internal tandem duplication; } \mathrm{MCL} \text {, mantle cell lym- } \\
\text { phoma; MDS, myelodysplastic syndrome; MDS/MPN, myelodysplastic/myelopro- } \\
\text { liferative neoplasm;TKD, tyrosine kinase domain. }\end{array}$} \\
\hline
\end{tabular}

\section{REFERENCES}

1. Jaffe ES, Campo E, Harris NL, et al. WHO Classification of Tumours of Haematopoietic and Lymphoid Tissues. WHO Publications Center; 2008.

2. Estey EH. Acute myeloid leukemia: 2014 update on risk-stratification and management. Am J Hematol. 2014;89(11):1063-1081.

3. Orazi A, Foucar K, Knowles DM, eds. Flow cytometry in the assessment of hematologic disorders. In: Knowles' Neoplastic Hematopathology. 3rd ed. Wolters Kluwer; 2013:110-145.

4. Craig FE, Foon KA. Flow cytometric immunophenotyping for hematologic neoplasms. Blood. 2008;111(8):3941-3967.

5. Johansson $U$, Bloxham D, Couzens $S$, et al. Guidelines on the use of multicolor flow cytometry in the diagnosis of haematological neoplasms: British Committee for Standards in Haematology. $\mathrm{Br} J$ Haematol. 2014;165(4):455-488. 
6. Pernick N. CD markers. Secondary CD markers. http://www.pathologyoutlines.com/cdmarkers.html

7. Grim KE BT, O'Malley DP, Weisee LM. Immunophenotypic markers useful in the diagnosis and classification of hematopoietic and lymphoid neoplasms. In: Orazi A, Foucar K, Knowles DM, eds. Knowles' Neoplastic Hematopathology. Wolters Kluwer; 2013:91-118.

8. Chan A, Enwere E, Johnson G, et al. Stains and molecular markers. Secondary stains and molecular markers. 2016. http://www.pathologyoutlines.com/stains.html

9. Foucar K, Reichard, K, Czuchlewski D. Bone Marrow Pathology. 3rd ed. American Society for Clinical Pathology; 2010.

10. Nihal M, Mikkola D, Wood GS. Detection of clonally restricted immunoglobulin heavy chain gene rearrangements in normal and lesional skin: analysis of the B cell component of the skin-associated lymphoid tissue and implications for the molecular diagnosis of cutaneous B cell lymphomas. J Mol Diagn. 2000;2(1):5-10.

11. Elenitoba-Johnson KS, Bohling SD, Mitchell RS, et al. PCR analysis of the immunoglobulin heavy chain gene in polyclonal processes can yield pseudoclonal bands as an artifact of low B cell number. J Mol Diagn. 2000;2(2):92-96.

12. Leonard DGB. Molecular Pathology in Clinical Practice: Genetics. Springer US; 2008:311.

13. Boehm TL, Werle A, Drahovsky D. Immunoglobulin heavy chain and T-cell receptor gamma and beta chain gene rearrangements in acute myeloid leukemias. Mol Biol Med. 1987;4(1):51-62.

14. Lee SC, Berg KD, Racke FK, et al. Pseudo-spikes are common in histologically benign lymphoid tissues. J Mol Diagn. 2000;2(3):145-152.

15. Caspersson T, Zech L, Johansson C. Differential binding of alkylating fluorochromes in human chromosomes. Exp Cell Res. 1970;60(3):315-319.

16. Jaffe ES, Harris NL, Vardiman JW, et al. Cytogenetic analysis and related techniques in hematopathology. In: Hematopathology. 1st ed. Elsevier Saunders; 2011:1058.

17. Streubel B, Simonitsch-Klupp I, Mullauer L, et al. Variable frequencies of MALT lymphoma-associated genetic aberrations in MALT lymphomas of different sites. Leukemia. 2004;18(10):1722-1726.

18. O'Keefe CL, Tiu R, Gondek LP, et al. High-resolution genomic arrays facilitate detection of novel cryptic chromosomal lesions in myelodysplastic syndromes. Exp Hematol. 2007;35(2):240-251.

19. Kallioniemi A, Kallioniemi OP, Sudar D, et al. Comparative genomic hybridization for molecular cytogenetic analysis of solid tumors. Science. 1992;258(5083):818-821.

20. Palanisamy N, Abou-Elella AA, Chaganti SR, et al. Similar patterns of genomic alterations characterize primary mediastinal large-B-cell lymphoma and diffuse large-B-cell lymphoma. Genes Chromosomes Cancer. 2002;33(2):114-122.

21. Joos S, Menz CK, Wrobel G, et al. Classical Hodgkin lymphoma is characterized by recurrent copy number gains of the short arm of chromosome 2 . Blood. 2002;99(4):1381-1387. 
22. Jinming S, Haipeng S. SNP array in hematopoietic neoplasms: a review. Microarrays. 2016;5(1):1. doi: 10.3390/microarrays5010001

23. Okamoto R, Ogawa $S$, Nowak D, et al. Genomic profiling of adult acute lymphoblastic leukemia by single nucleotide polymorphism oligonucleotide microarray and comparison to pediatric acute lymphoblastic leukemia. Haematologica. 2010;95(9):1481-1488.

24. Mardis ER. Next-generation sequencing platforms. Annu Rev Anal Chem. 2013;6:287-303.

25. Chin EL, da Silva C, Hegde M. Assessment of clinical analytical sensitivity and specificity of next-generation sequencing for detection of simple and complex mutations. BMC Genet. 2013;14:6.

26. Swerdlow SH, Campo E, Pileri SA, et al. The 2016 revision of the World Health Organization (WHO) classification of lymphoid neoplasms. Blood. 2016;127(20):2375-2390. doi: 10.1182/blood-2016-01-643569

27. Arber DA, Orazi A, Hasserjian R, et al. The 2016 revision to the World Health Organization (WHO) classification of myeloid neoplasms and acute leukemia. Blood. 2016;127:2391-2405.

28. Darzynkiewicz Z, Halicka HD, Zhao H. Analysis of cellular DNA content by flow and laser scanning cytometry. Adv Exp Med Biol. 2010; 676:137-147. 